\title{
Optimal Rule Selection Scheme using Concept Relationship Analysis
}

\author{
R.Renuga Devi \\ Department of Computer Science and Applications \\ K S R College of Arts and Science \\ Tiruchengode
}

\author{
R. Manavalan \\ Department of Computer Science and Applications \\ K S R College of Arts and Science \\ Tiruchengode
}

\begin{abstract}
In Data Mining, the Association rule mining is used to retrieve the recurrent item sets. Apriori algorithm is mainly used to mine association rules. In that, rule reduction is required for efficient decision-making system. Knowledge based rule reduction schemes are used to filter the interested rules. In the existing system rule validation is not provided. Quantitative attributes are not considered in the post-mining scheme. Weighted rule mining scheme is not supported. This paper proposes Weighted Rule mining approach to perform post mining on derived rules with ontology support.Post mining schemes are used to filter consequent rules. Based on the Support and confidence values, the interested rules are selected rules and the same is used for the decision making process. Here, rule-mining scheme is improved to handle quantitative attributes. The WARM method is improved with validation methods. Then weighted rule mining and filtering process can be incorporated with the ARIPSO scheme. And also the rank based concept relationship analysis can be provided to improve the post mining process. Ontology based Association rule mining and Ontology based weighted Rule mining comparative analysis are focused.
\end{abstract}

\section{Keywords}

Classification, association rule mining, Ontologies, Weighted rule mining, Post mining, ARIPSO.

\section{INTRODUCTION}

Association rule mining is one of the most important tasks in Knowledge Discovery in Databases. From the given set of items in transaction databases, it looks at discovering implicative tendencies that can be important information for the decision-maker.

An association rule is defined as the implication $X \rightarrow Y$, defined by two interestingness measures - support and confidence, here $\mathrm{X}$ and $\mathrm{Y}$ are the sets of items and $\mathrm{X} \cap \mathrm{Y}$ $=\varnothing$.

On the other hand, most of the existing postprocessing methods are based on statistical information in the database. So, the rule interestingness are fully depends on user knowledge, these methods does not give assurance that interesting rules will be extracted. For example, if the user looks for unanticipated rules, have to check all the well-known rules should be pruned. Or, if the user wants to focus on particular schemas of rules, only this subset of rules should be selected. Strong interactivity with the user should be there in the postprocessing methods. The representation of user knowledge is an important thing. The knowledge is represented in a flexible, communicative, and perfect formalism, the rule selection is efficient. In the Semantic
Web1 field, ontology is used to express the complexity of the user knowledge, and several specification languages were proposed.

This paper proposes a new interactive post processing approach, ARIPSO (Association Rule Interactive postProcessing using Schemas and Ontologies) to prune and filter discovered rules. Here, we propose the use Domain Ontologies in order to support the integration of user knowledge in the postprocessing task. Next, we introduce Rule Schema method by extending the specification language proposed by Liu et al. In addition, an interactive and iterative framework is designed to help the user throughout the analyzing task. The interactivity of our approach relies on a set of rule mining operators defined over the Rule Schemas In order to express the actions that the user can perform. The Simplest way to do this is simply to download the template, and replace the content with your own material.

\section{RELATED WORKS}

\subsection{Concise Representations of Recurrent Itemsets}

Interestingness measures represent metrics in the process of accruing dependencies and implications between database items, and express the strength of the pattern association relationship.

Since frequent itemset creation is considered as a costly operation, mining frequent closed itemsets was proposed in order to reduce the number of frequent itemsets. For example, an itemset $\mathrm{X}$ is denoted as closed frequent itemset if $\notin$ itemset $X^{\prime} \subseteq X$ so that $\mathrm{t}(\mathrm{X})=\mathrm{t}\left(\mathrm{X}^{\prime}\right)$. Thus, the number of frequent closed itemsets generated is reduced in comparison with the number of frequent itemsets.

The CLOSET algorithm was proposed and provides the new efficient method for mining closed itemsets. CLOSET uses a novel frequent pattern tree (FP-tree) structure, which is a condensed representation of all the transactions in the database. It uses a recursive divideand-conquer and database projection approach another solution for the reduction of the number of frequent itemsets is mining maximal frequent itemsets [1]. The MAFIA algorithm was proposed, and it based on depthfirst traversal and several pruning methods as Parent Equivalence Pruning (PEP), FHUT, HUTMFI, or Dynamic Recording. The main drawback of extracting maximal frequent itemsets is the loss of information because the subset frequency is not available and generating rules is not possible. 


\subsection{Redundancy Reduction}

Creating all association rules that satisfy the confidence threshold is the main problem. Zaki and Hsiao used recurrent closed itemsets in the CHARM algorithm produce all frequent closed itemsets. They used itemsettid to set search tree and pursued with the aim of generating a small nonredundant rule set [2].

Pasquier et al. proposed the Close algorithm to extract association rules. It is based on a new mining method: pruning of the closed set pattern in order to extract frequent closed itemsets. Li [3] proposed optimal rules sets, defined with respect to interestingness metric. An optimal rule set contains all rules except those with no greater interestingness than one of its more general rules. Hahsler et al. [4] were interested in generating association rules from arbitrary sets of itemsets. This makes the user to propose a set of Itemsets and to combine another set generated by a data mining tool. Toivonen et al. proposed a new technique for redundancy reduction based on rule covers. The idea of rule cover is defined as the subset of a rule set describing the same database transaction set as the rule set.

\subsection{User-Driven Association Rule Mining}

Interestingness measures were proposed in order to discover only those association rules that are interesting according to these measures. They have been divided into objective measures and subjective measures. Objective measures are based on data structure. Many survey papers review and compare the objective measure definitions and properties [5]. Unfortunately, being restricted to data evaluation, the objective measures are not enough to reduce the number of extracted rules and to confine the interesting ones. Several methods for integrating user knowledge have been proposed.

The key finding and deviation notions were suggested. Grouped in findings, deviations represent the difference between the actual and the expected values. KEFIR defines interestingness of a key finding in terms of the estimated benefits, and potential savings of taking corrective actions that restore the deviation back to its expected value. These corrective actions are specified in advance by the domain expert for various classes of deviations.

Later, Klemettinen et al proposed templates to describe the form of interesting rules and not interesting rules. The idea of using templates for association rule extraction was reused.

Imielinski et al proposed a query language for association rule pruning based on SQL, called M-SQL. It allows imposing constraints on the condition and/or the consequent of the association rules.

Another related approach was proposed by An et al. Where the authors introduced domain knowledge in order to prune and summarize discovered rules. In 2007, a new methodology was proposed in [6] to prune and organize rules with the same consequent. The authors suggested transforming the database in an association rule base in order to extract second-level association rules, Called metarules.

\subsection{Ontologies in Data Mining}

In knowledge engineering and Semantic Web fields, ontologies have interested researchers since their first proposition in the philosophy branch by Aristotle. Ontologies have evolved over the years from controlled vocabularies to thesauri and later, to taxonomies.

Ontology was defined by Gruber as a formal, explicit specification of a shared conceptualization. By conceptualization, we understand here an abstract model of some phenomenon described by its important concepts. The formal notion denotes the idea that machines should be able to interpret ontology. Moreover, explicit refers to the transparent definition of ontology elements. Finally, shared outlines that Ontology brings together some knowledge common to a certain group, and not individual knowledge.

In the year of 2000, Ontologies introduced in data mining for the first time, and it can be used in several ways [7]: Such as, Meta data Ontologies, Domain and Background Knowledge Ontologies and Ontologies for Data Mining Process. Metadata Ontologies used to describe the construction process of items. Background Knowledge Ontologies categorize domain knowledge and play important roles at various levels of the knowledge discovery process. In the Data Mining Process, the Ontologies codify mining process description and select the most appropriate task based on the given problem. In this paper, we mainly focus on Domain and Background Knowledge Ontologies. The first idea of using Domain Ontologies was introduced by Srikant and Agrawal with the concept of Generalized Association Rules (GAR). The authors proposed taxonomies of mined data in order to generalize/specify rules.

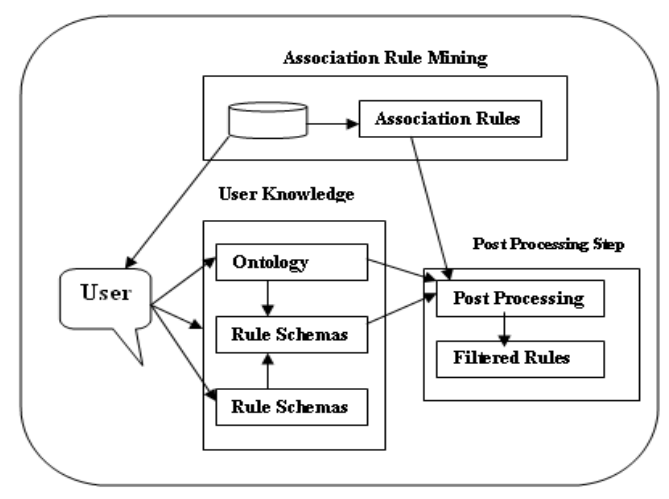

Fig.1 Framework Description

\section{DESCRIPTION OF THE ARIPSO FRAMEWORK}

The proposed method is consists of two main parts (as shown in Fig.1). First, the knowledge base allows formalizing user knowledge and goals. Domain knowledge offers a general view over user knowledge in database domain, and user expectations express the prior user knowledge over the discovered rules. Second, the postprocessing operation consists of applying iteratively a set of filters over extracted rules in order to extract interesting rules: minimum improvement constraint filter, item-relatedness filter, rule schema filters/pruning. 
The novelty of this approach resides in supervising the knowledge discovery process using two different conceptual structures for user knowledge representation: one or several ontologies and several rule schemas generalizing general impressions, and proposing an iterative process.

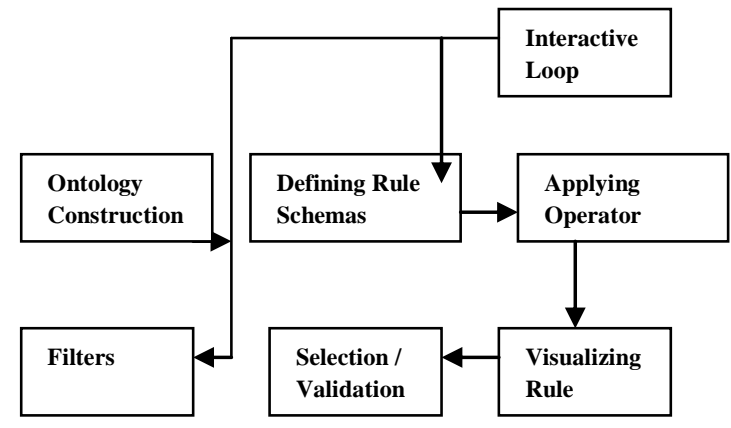

Fig.2 Interactive process

\subsection{Interactive Postmining Process}

The ARIPSO framework proposes an interactive process of rule discovery, shown in Fig.2. Based on the user's feedback, the user is able to revise their expectations in function of intermediate results. Several steps are suggested to the user in the framework. Such as, Ontology construction is starting from the database, and from the existing Ontologies, the user can creates ontology on database items. The Rule schema defining process, the user can express their ideas and expectations relating to the association rules that they wants to find. Choosing the correct operators to be applied over the rule schemas created, and then, applying the operators. Visualizing methods is mainly used to produce the filtered association rules are proposed to the user. Selection and validation process starting from the basic results, the user can examine the results or he/she can alter his/her information. We propose two filters already existing in the literature and detailed. It can be applied over rules whenever the user needs them with the main goal of reducing the number of rules, and the interactive loop permits to the user to revise the information that he/she proposed. Thus, he/she can modify the rule schemas, or he/she can change the operators. Moreover, in the interactive loop, the user could decide to apply one of the two predefined filters.

\subsection{Enhancing Universal Impressions with Ontologies}

To improving association rule selection, we recommend a new rule filtering model, called Rule Schemas (RS). A rule schema is a rule-like formalism, the user hope in terms of interesting/obvious rules. As a result, Rule Schemas act as a rule grouping, defining rule families. The Rule Schema formalism is based on the specification language introduced by Liu et al. The model proposed by Liu et al. is described using elements from an item taxonomy allowing as an organization of database attributes. This item taxonomy has many advantages, such as the representation of user expectations is more general, and filtered rules are more interesting for the user.On the other hand, taxonomy of items might not be enough. The user wants to use concepts, when compared to generalized concepts it must be more meaningful and accurate. This is why we have considered that the use of ontologies would be more appropriate. Ontology includes the features of taxonomies but adds more representation power. In taxonomy, the means for subject description consist essentially of one relationship: the subsumption relationship used to build the hierarchy. The set of items is opened, but the language used to describe them is closed [12] by using a single relationship. The taxonomy is simply a hierarchical group or classification of items in a domain. On the converse, an ontology is a specification of several characteristics of a domain, defined using an open terminology.

In addition, for a domain expert it is difficult to know about the support and confidence values for each rule schema proposed, because of their statistical definition. So we consider that using Precise Knowledge in user expectation representation might be useless. So we propose a method to improve only two of the three representations introduced: General Impressions and Reasonably Precise Concepts.

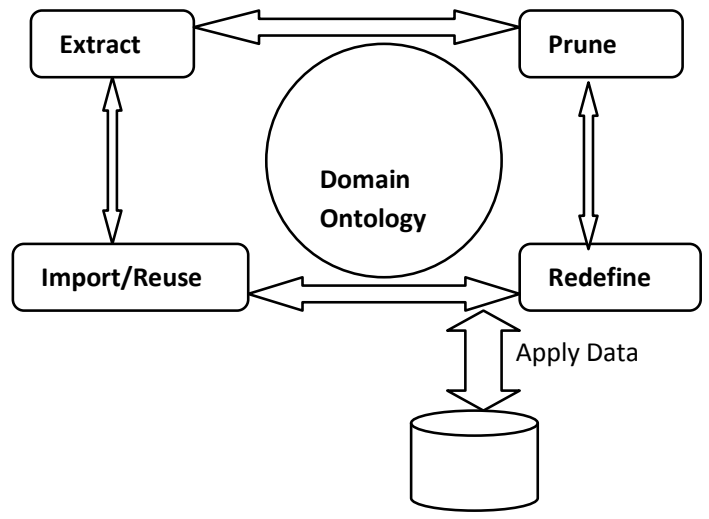

Fig.3 Ontology Learning Process

\subsection{Ontology Description}

Domain knowledge, defined as the user information concerning the database, is described in our framework using Ontologies. Fig.3. shows the steps, which is involved in the Ontologies learning process. In this scenario, it is fundamental to connect ontology concepts $\mathrm{C}$ of $\mathrm{O}=\{\mathrm{C}, \mathrm{R}, \mathrm{I}, \mathrm{H}, \mathrm{A})$ to the database, each one of them being connected to one/several items of I. To this end, we consider three types of concepts: leaf-concepts, generalized concepts from the subsumption relation (<) in $\mathrm{H}$ of $\mathrm{O}$, and restriction concepts proposed only by Ontologies. In order to proceed with the definition of each type of concepts, let us remind that a set of items in a database is defined as $I=\{i 1, i 2, \ldots$ in $\}$.

The leaf-concepts $(\mathrm{C} 0)$ are defined as

$$
C_{0}=\left\{c_{0} \in C \mid \notin C, c^{\prime} \leq c_{0}\right\}
$$

They are connected in the easiest way to database - each concept from $\mathrm{C} 0$ is associated to one item in the database:

$$
f_{0}: C_{0} \rightarrow I, \forall c_{0} \in C_{0}, \exists i \in I, i=f_{0}\left(c_{0}\right)
$$

Generalized concepts (C1) are described as the concepts that subsume other concepts in the ontology. A 
generalized concept is connected to the database through its subsumed concepts[10]. This means that, recursively, only the leaf-concepts subsumed by the generalized concept contribute to its database connection:

$$
\begin{aligned}
& f: C_{1} \rightarrow 2^{I} \\
& \forall c_{1} \in C_{1}, f\left(c_{1}\right)=\bigcup_{c_{0} \in C_{0}}\left\{i=f_{0}\left(c_{0}\right) \mid c_{0} \leq c_{1}\right\}
\end{aligned}
$$

Restriction concepts are described using logical expressions defined over items and are organized in the C2 subset. In a first attempt, we base the description of the concepts on restrictions over properties available in description logics. Thus, the restriction concept defined could be connected to a disjunction of items[14].

\subsection{Operations on Rule Schemas}

The rule schema filter is based on operators applied over rule schemas allowing the user to perform several actions over the discovered rules. We propose two important operators: pruning and filtering operators. The filtering operator is composed of three different operators: conforming, unexpectedness, and exception [13]. We propose to reuse the operators proposed by Liu et al.: conforming and unexpectedness, and we bring two new operators in the postprocessing task: pruning and exceptions.

\subsection{Filters}

In order to reduce the number of rules, three filters incorporate the framework: operators applied over rule schemas, minimum improvement constraint filter, and item relatedness filter. Minimum improvement constraint filter (MICF) selects only those rules whose confidence is greater with minimum than the confidence of any of its simplifications. We can note that the last two rules are the simplifications of the first one. The theory of Bayardo et al. tells us that the first rule is interesting only if its confidence improves the confidence of all its simplifications. In our case, the first rule does not improve the confidence of 90 percent of the best of its simplifications, so it is not considered as an interesting rule, and it is not selected.

The item-relatedness filter (IRF) was proposed by Shekar and Natarajan. Starting from the idea that the discovered rules are generally obvious, they introduced the idea of relatedness between items measuring their semantic distance in item taxonomies. This measure computes the relatedness of all the couples of rule items [9]. We can notice that we can compute the relatedness for the items of the condition or/and the consequent, or between the condition and the consequent of the rule.

\section{WEIGHTED ASSOCIATION RULE MINING}

Association rule was first introduced to introduce the support-confidence measurement framework and reduced association rule mining to the discovery of frequent item sets. Later a fast algorithm [1], Apriori, was proposed. Much effort has been dedicated to the association rule mining problem. Many algorithms have been proposed to extract the rules efficiently. These algorithms severely follow the classical dimension framework and produce the same results once the minimum support and minimum confidence are given. WARM generalizes the traditional model to the case where items have weights. Ram Kumar et al. introduced weighted support of association rules based on the costs assigned to both items as well as transactions. A WIS algorithm was proposed to derive the rules that have a weighted support larger than a given threshold. Ca et al. defined weighted support in a similar way except that they only took item weights into account [11]. The definition broke the downward closure property. As a result, the proposed mining algorithm became more complicated and time consuming. Tao et al provided another definition to retain the "weighted downward closure property."

Wang and Su proposed a new approach on item ranking [3]. A directed graph is created where nodes denote items and links represent association rules. A generalized version of HITS is applied to the graph to rank the items, where all nodes and links are allowed to have weights. However, the model has a limitation that it only ranks items but does not provide a measure like weighted support to evaluate an arbitrary item set. Anyway, it may be the first successful attempt to apply link-based models to association rule mining [8].

\section{CONCEPT RELATIONSHIP BASED RULE SELECTION MODEL}

The association rule mining methods are used to mine frequent patterns. Minimum support and minimum confidence values are used to find interested rules. The post mining operations are useful to filter detected rules. Redundant rules are filtered in the post mining process. The ontology maintains the concept relationship between the terms. The attribute name relationships are analyzed with the ontology details. The ontology methods are used to support post-mining operations. The rules are filtered using the ontology relationships. The main objectives of the system is to

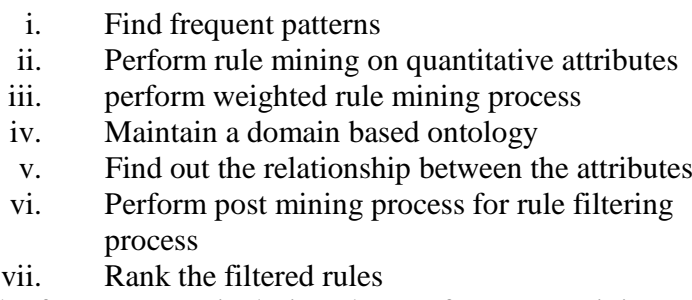
The future system is designed to perform post mining on derived rules. ARIPSO scheme is improved with validation methods. Weighted rule mining and filtering process can be integrated with the ARIPSO scheme. Rank based concept relationship analysis can be provided to improve the post mining process. The system is designed to perform rule mining and rule selection process. Ontology is used to reduce the rules based on concept relationships. Weighted rule mining scheme is also integrated with the system. Rule validation process is included to verify the mined rules. The proposed system consists of five stages and it shown in Figure 5.1. 


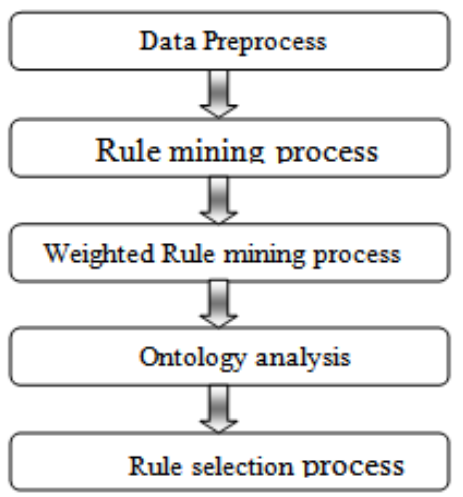

Fig.4 Different stages of Proposed System

Data preprocess module is designed to perform noise elimination and candidate set preparation tasks. Ontology construction and attribute analysis are performed under ontology analysis module. Interested rule selection is carried out under rule mining process. Weighted rule mining is applied with attribute weight values. Post mining operations are carried out under the rule selection process.

\subsection{Data Preprocess}

The data preprocess module is used to normalize the data values. Noise elimination process is performed to reduce redundant data values. Attribute names and values are extracted to build candidate sets. Frequency estimation is done for each candidate set values.

\subsection{Rule Mining Process}

The association rule mining tasks are carried out under the rule mining process. Candidate generation is performed with attribute names and attributes values for each transaction. The item sets are prepared from the candidate set information. Frequency values are estimated for each item. The support and confidence values are estimated for all items.

An association rule is an implication $\mathrm{X} \rightarrow \mathrm{Y}$, where $\mathrm{X}$ and $Y$ are two item sets and $X \cap Y=\varnothing$. The $X$ is called the antecedent of the rule, and the $\mathrm{Y}$ is called the consequent.

Support Value: The support of the rule, defined as $\operatorname{supp}(X \rightarrow Y)=\operatorname{supp}(X U Y)=|t(X U Y)|$, is the ratio of the number of transactions containing $X$ U Y. If supp $(\mathrm{X} \rightarrow \mathrm{Y})=\mathrm{s}, \mathrm{s} \%$ of transactions contains the itemset $\mathrm{X}$ UY.

Confidence Value: The confidence of the rule, defined as $\operatorname{conf}(\mathrm{X} \rightarrow \mathrm{Y})=\operatorname{supp}(\mathrm{X} \rightarrow \mathrm{Y}) / \operatorname{supp}(\mathrm{X})=\operatorname{supp}(\mathrm{X} \mathrm{U}$ $\mathrm{Y}) / \operatorname{supp}(\mathrm{X})$, is the ratio (c \%) of the number of transactions that, containing $\mathrm{X}$, contain also $\mathrm{Y}$.

The interested rule selection process is carried out on the estimated support and confidence values. Minimum support and minimum confidence values are used to filter the relevant rules.

\subsection{Weighted Rule Mining Process}

The association rule mining process used frequency values for mining process. It is not suitable for all types of transactions. So that, Weight values is used in the rule mining process. Weight and frequency values are used to estimate weighted support and weighted confidence values. The minimum support and minimum confidence values are used to filter the weighted rules.

\subsection{Ontology Analysis}

The ontology is a repository used to maintain the relationship between the concepts and terms. The ontology is maintained as XML documents. The resource description framework is used to manage ontology values. The ontology is used to analyze the attribute relationship. The transaction table attribute names are analyzed with ontology elements. The relationship and their levels are extracted from the ontology analysis.

\subsection{Rule Selection Process}

The rule selection process is done with ontology analysis and pruning model. The user assisted rule selection is also carried out to filter the rules. The system selects the rules under the post mining process. The ontology is used extract relationship between the attributes. The rules are ranked with reference to the concept weight values.

\section{EXPERIMENTAL ANALYSIS}

The algorithms discussed in previous section are implemented using java. The experiment is conducted over a mushroom dataset, which is a benchmark dataset, available in the UCI (University of California Irwin) machine learning repository. The twenty four features and 7668 transactions are maintained in the mushroom datasets. Each transaction is represented by 24 features which are formed by using general and structural properties of mushroom. The concept relationships are maintained in the Ontology. In the Ontology, three types of relationship information are maintained, such as synonym, meronym and hypernym. Ontology is constructed using XML and the same is used for analysis of the attribute relationship in the dataset. The performance of the optimal rule selection process is carried out with rule mining and rule selection parameters, such as support and confidence level values. Once the rules are constructed, then the interested rules are mined by using proposed methods. The ranges of support and confidence values are from 0.1 to 1 . In this paper, the support value and confidence level value are set to 0.1 and 0.2 respectively to extract the interested rules.

The selection of rules is based on attribute relationship and weight values. Association rule mining and weighted rule mining results are analyzed for measuring the mined rules. The number of rules mined by ARM and WRM is recorded in the table.1. The fig. 5 shows the performance of rule mining analysis.

Table.1. Rule Mining Analysis - ARM Vs WARM

\begin{tabular}{|c|c|c|c|}
\hline Transactions & $\begin{array}{c}\text { Total } \\
\text { Rules }\end{array}$ & $\begin{array}{c}\text { Rule } \\
\text { Count }\end{array}$ & $\begin{array}{c}\text { Weighted } \\
\text { Rule Count }\end{array}$ \\
\hline 500 & 1762 & 876 & 892 \\
\hline 1000 & 3216 & 1538 & 1530 \\
\hline 1500 & 4437 & 1892 & 1815 \\
\hline 2000 & 6176 & 2363 & 2287 \\
\hline 2500 & 7943 & 2842 & 2729 \\
\hline
\end{tabular}


Table.3. Rule Selection Analysis - WARM Vs OBS

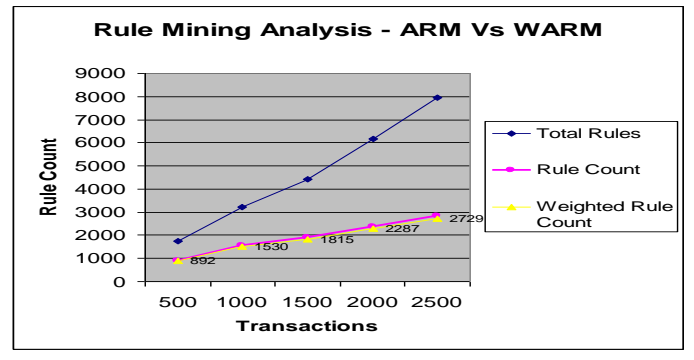

Fig.5 Rule Mining Analysis - ARM Vs WARM

The results in the table.1 shows that weighted Association rule mining process select minimum number of rules compared to Association Rule Mining. Since weighted rule mining uses the frequency and weight values.

Ontology based optimal rule selection process is applied on the results of association rule mining and weighted association rule mining process separately. The application of ontology based optimal rule selection considers as post mining process and their results are reported in table. 2 and table. 3 respectively. Then the performance the rules selection is depicted in the fig. 6 and fig.7.

Table.2. Rule Selection Analysis - ARM Vs OBS

\begin{tabular}{|c|c|c|}
\hline Transactions & Rule Count & Optimal Rules \\
\hline 500 & 876 & 18 \\
\hline 1000 & 1538 & 33 \\
\hline 1500 & 1892 & 38 \\
\hline 2000 & 2363 & 45 \\
\hline 2500 & 2842 & 54 \\
\hline
\end{tabular}

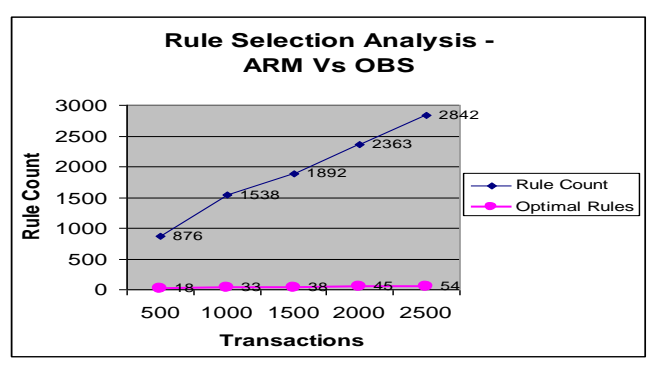

Fig.6 Rule Selection Analysis - ARM Vs OBS

\begin{tabular}{|c|c|c|}
\hline Transactions & $\begin{array}{c}\text { Weighted Rule } \\
\text { Count }\end{array}$ & $\begin{array}{c}\text { Optimal } \\
\text { Rules }\end{array}$ \\
\hline 500 & 892 & 14 \\
\hline 1000 & 1530 & 26 \\
\hline 1500 & 1815 & 32 \\
\hline 2000 & 2287 & 39 \\
\hline 2500 & 2729 & 45 \\
\hline
\end{tabular}

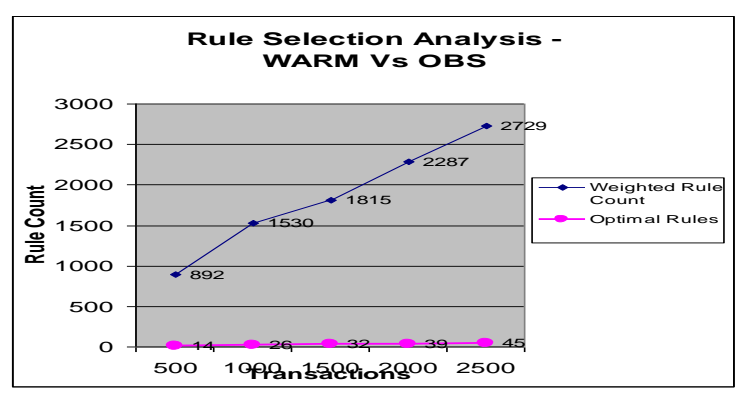

Fig.7 Rule Selection Analysis - WARM Vs OBS

The number of optimal rule selected from the results of association rule mining and weighted rule mining models are offered in table shown in table.4., for comparative analysis and their performance is represented in fig.8.

Table.4. Comparative Analysis of OBS-ARM Vs OBS- WARM

\begin{tabular}{|c|c|c|}
\hline Transactions & OBS-ARM & OBS- WARM \\
\hline 500 & 18 & 14 \\
\hline 1000 & 33 & 26 \\
\hline 1500 & 38 & 32 \\
\hline 2000 & 45 & 39 \\
\hline 2500 & 54 & 45 \\
\hline
\end{tabular}

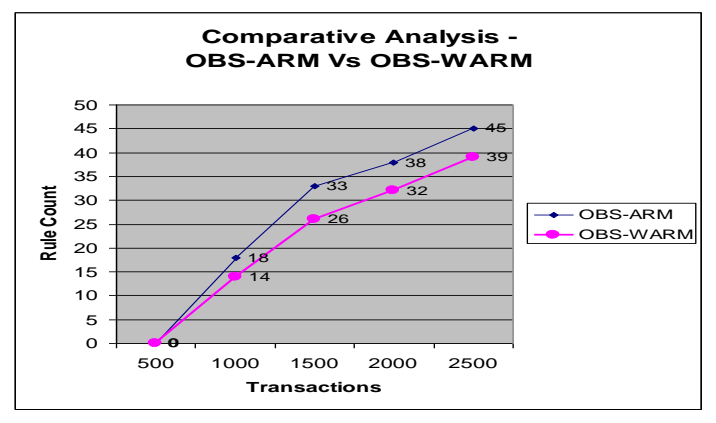

Fig.8. Comparative Analysis of OBS-ARM Vs OBSWARM. 
Table.5. Average Result Analysis

\begin{tabular}{|c|c|c|c|c|c|}
\hline 氖 & 吾 $\frac{\tilde{E}}{E}$ & $\sum_{\approx}^{\approx}$ & 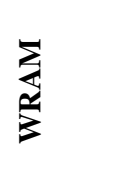 & 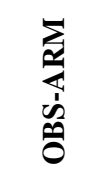 & 㐫 \\
\hline 500 & 1762 & $49.71 \%$ & $50.62 \%$ & $2.04 \%$ & $1.56 \%$ \\
\hline 1000 & 3216 & $47.82 \%$ & $47.57 \%$ & $2.14 \%$ & $1.69 \%$ \\
\hline 1500 & 4437 & $42.64 \%$ & $40.90 \%$ & $2.0 \%$ & $1.76 \%$ \\
\hline 2000 & 6176 & $38.26 \%$ & $37.03 \%$ & $1.90 \%$ & $1.70 \%$ \\
\hline 2500 & 7943 & $35.64 \%$ & $34.22 \%$ & $1.90 \%$ & $1.64 \%$ \\
\hline \multicolumn{2}{|c|}{ AVERAGE } & $42.81 \%$ & $42.06 \%$ & $1.99 \%$ & $1.67 \%$ \\
\hline
\end{tabular}

The table.5 shows that, the Association rule mining method filters average of $42.81 \%$ rules from the total number of rules. Similarly Weighted association rule mining filters average of $42.06 \%$ rules from the total rules. Here, while applying ontology based selection method, OBS-ARM selects the average of $1.99 \%$ of optimal rules from the filtered rules where as the OBSWARM selects the average of $1.67 \%$ of optimal rules which is $0.32 \%$ lesser than OBS-ARM. Therefore the proposed model OBS-WARM selects most frequent items are efficiently and produce better results with high rule filtering accuracy levels.

\section{CONCLUSION}

This paper addresses the problem of selecting interesting association rules throughout huge volumes of discovered rules. The major contributions of the paper are stated below. First, we propose a method to integrate user knowledge in association rule mining using two different types of formalism: Ontologies and Weighted Association Rule mining. On the one hand, domain Ontologies improves the integration of user domain knowledge concerning the database field in the postprocessing step. On the other hand, we propose a method called OBS-WARM to select most optimal rules from the item sets. Weight information is used for the rule mining process. Attribute relationship are identified by using Ontology. The Proposed OBS-WARM approach produces the better results when compared to OBS-ARM.

By applying our new approach over a voluminous questionnaire database, allowed the integration of domain expert knowledge in the postprocessing step in order to reduce the number of rules to several dozens or less. Moreover, the quality of the filtered rules was validated by the expert throughout the interactive process.

\section{REFERENCES}

[1] D. Burdick, M. Calimlim, J. Flannick, J. Gehrke, and T. Yiu, "Mafia: A Maximal Frequent Itemset
Algorithm," IEEE Trans. Knowledge and Data Eng., vol. 17, no. 11, pp. 1490-1504, Nov. 2005.

[2] M.J. Zaki, "Generating Non-Redundant Association Rules," Proc. Int'l Conf. Knowledge Discovery and Data Mining, pp. 34-43, 2000.

[3] J. Li, "On Optimal Rule Discovery," IEEE Trans. Knowledge and Data Eng., vol. 18, no. 4, pp. 460471, Apr. 2006

[4] M. Hahsler, C. Buchta, and K. Hornik, "Selective Association Rule Generation," Computational Statistic, vol. 23, no. 2, pp. 303-315, Kluwer Academic Publishers, 2008.

[5] F. Guillet and H. Hamilton, Quality Measures in Data Mining. Springer, 2007.

[6] A. Berrado and G.C. Runger, "Using Metarules to Organize and Group Discovered Association Rules," Data Mining and Knowledge Discovery, vol. 14, no. 3, pp. 409-431, 2007.

[7] H. Nigro, S.G. Cisaro, and D. Xodo, Data Mining with Ontologies: Implementations, Findings and Frameworks. Idea Group, Inc., 2007.

[8] X. Zhou and J. Geller, "Raising, to Enhance Rule Mining in Web Marketing with the Use of an Ontology," Data Mining with Ontologies: Implementations, Findings and Frameworks, pp. 1836, Idea Group Reference, 2007.

[9] A. Bellandi, B. Furletti, V. Grossi, and A. Romei, "Ontology- Driven Association Rule Extraction: A Case Study," Proc. Workshop Context and Ontologies: Representation and Reasoning, pp. 110, 2007.

[10] A.C.B. Garcia and A.S. Vivacqua, "Does Ontology Help Make Sense of a Complex World or Does It Create a Biased Interpretation?" Proc. Sensemaking Workshop in CHI '08 Conf. Human Factors in Computing Systems, 2008.

[11] A.C.B. Garcia, I. Ferraz, and A.S. Vivacqua, "From Data to Knowledge Mining," Artificial Intelligence for Eng. Design, Analysis and Manufacturing, vol. 23, pp. 427-441, 2009.

[12] L.M. Garshol, "Metadata? Thesauri? Taxonomies? Topic Maps Making Sense of It All," J. Information Science, vol. 30, no. 4, pp. 378-391, 2004.

[13] I. Horrocks and P.F. Patel-Schneider, "A Proposal for an owl Rules Language," Proc. 13th Int'l Conf. World Wide Web, pp. 723-731, 2004.

[14] M.-A. Storey, N.F. Noy, M. Musen, C. Best, R. Fergerson, and N. Ernst, "Jambalaya: An Interactive Environment for Exploring Ontologies," Proc. Seventh Int'l Conf. Intelligent User Interfaces (IUI '02), pp. 239-239, 2002.

[15] Claudia Marinica and Fabrice Guillet "KnowledgeBased Interactive Postmining of Association Rules Using Ontologies" IEEE Transactions on Knowledge and Data Engineering, Vol. 22, No. 6, June 2010. 